Provided for non-commercial research and education use. Not for reproduction, distribution or commercial use.

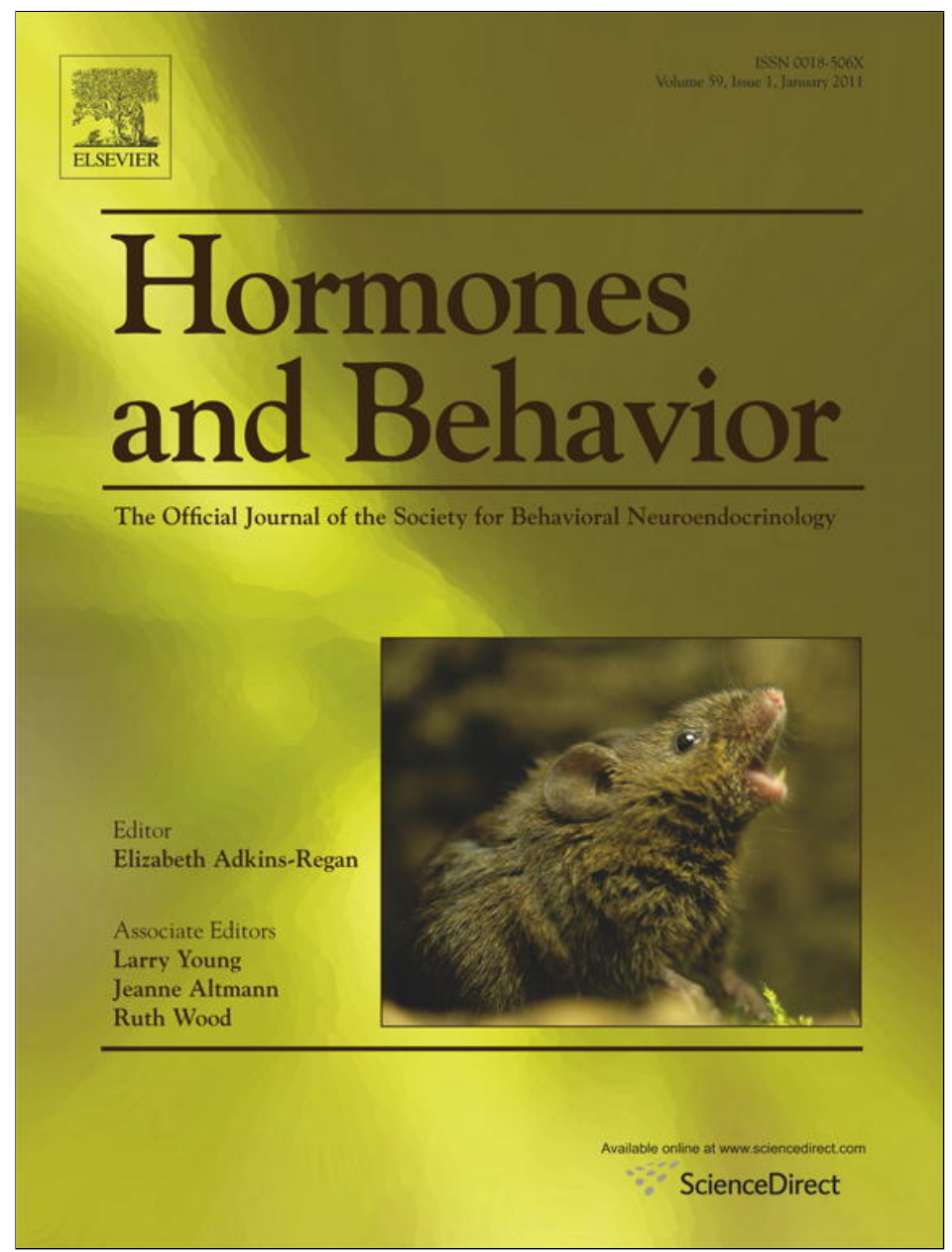

This article appeared in a journal published by Elsevier. The attached copy is furnished to the author for internal non-commercial research and education use, including for instruction at the authors institution and sharing with colleagues.

Other uses, including reproduction and distribution, or selling or licensing copies, or posting to personal, institutional or third party websites are prohibited.

In most cases authors are permitted to post their version of the article (e.g. in Word or Tex form) to their personal website or institutional repository. Authors requiring further information regarding Elsevier's archiving and manuscript policies are encouraged to visit:

http://www.elsevier.com/copyright 


\title{
Potential contribution of prenatal estrogens to the sexual differentiation of mate preferences in mice
}

\author{
Olivier Brock, Julie Bakker* \\ GIGA-Neurosciences, University of Liege, Avenue de l'Hôpital 1 (B36), 4000 Liege, Belgium
}

\section{A R T I C L E I N F O}

\section{Article history:}

Received 23 June 2010

Revised 14 October 2010

Accepted 18 October 2010

Available online 26 October 2010

\section{Keywords:}

$\alpha$-fetoprotein knockout

Sexual differentiation

Estradiol

Mate preference

\begin{abstract}
A B S T R A C T
The neural mechanisms controlling sexual behavior are sexually differentiated by perinatal actions of gonadal hormones. We recently observed using female mice deficient in alpha-fetoprotein (AFP-KO) and which lack the protective actions of AFP against maternal estrogens, that exposure to prenatal estrogens completely defeminized their potential to show lordosis behavior in adulthood. Therefore, we determined here whether mate preferences were also affected in female AFP-KO mice. We observed a robust preference for an estrous female over an intact male in female AFP-KO mice, which were ovariectomized in adulthood and subsequently treated with estradiol and progesterone, whereas similarly treated WT females preferred the intact male over the estrous female. Gonadally intact WT males preferred the estrous female over the male, but only when visual cues were blocked by placing stimulus animals behind opaque partitions. Furthermore, when given the choice between an intact male and a castrated male, WT females preferred the intact male, whereas AFP-KO females showed no preference. Finally when given the choice between an estrous female and an ovariectomized female, WT males preferred the estrous female whereas AFP-KO females preferred the ovariectomized female or showed no preference depending on whether they could see the stimulus animals or not. Taken together, when AFP-KO females are tested under estrous conditions, they do not show any maledirected preferences, indicating a reduced sexual motivation to seek out the male in these females. However, they do not completely resemble males in their mate preferences suggesting that the male-typical pattern of mate preferences is not solely organized by prenatal estrogens.
\end{abstract}

(c) 2010 Elsevier Inc. All rights reserved.

\section{Introduction}

When in breeding condition, male and female mammals usually seek out and mate with opposite sex conspecifics. It has been well established that the neural mechanisms controlling mate preference are sexually differentiated by the perinatal actions of sex steroid hormones (Bakker, 2003). Thus, a female-directed preference develops in male rats under the early (perinatal) influence of estradiol derived from neural aromatization of testosterone (Bakker et al., 1993, 1996a,b). Male rats treated neonatally with 1,4,6-androstatriene-3,17-dione (ATD), a specific inhibitor of the aromatase enzyme, failed to show a preference for an estrous female when given a choice between an estrous female and a sexually active male in a three compartment box, whereas normal males clearly preferred the vicinity of the estrous female. In fact, such neonatally ATD-treated male rats preferred the vicinity of the sexually active male, in particular following estradiol treatment in adulthood (Bakker et al.,

\footnotetext{
* Corresponding author. Fax: +324366 5953

E-mail address: jbakker@ulg.ac.be (J. Bakker).
}

1993, 1996a,b) suggesting that their mate preferences had not been masculinized.

We showed using aromatase knockout (ArKO) mice, which carry a targeted mutation in the Cyp19 gene (Bakker et al., 2002a) and as a result cannot convert androgens into estrogens, that in the mouse, like in the rat, female-directed preferences seem to develop under the influence of estrogens (Bakker et al., 2004). Gonadally intact male ArKO mice failed to show a preference for an estrous female when provided with volatile body odors from an estrous female and a sexually active male in a Y-maze (Bakker et al., 2002a), whereas wildtype (WT) males preferred to investigate the estrous female box. Adult treatment of male ArKO mice with estradiol failed to induce a female-directed preference suggesting that mate preferences develop perinatally in male mice under the influence of estrogens (Bakker et al., 2004). Similar results have been obtained in male mice carrying a mutation in the estrogen receptor alpha gene (ERalphaKO; Wersinger and Rissman, 2000). Such ERalphaKO males failed to show a preference for an estrous female when given the choice between an anesthetized estrous female and an anesthetized intact male, further confirming the pivotal role of estrogens and ER in the development of female-directed preferences.

Interestingly, we recently observed female-typical (i.e., maledirected) preferences in female mice carrying a mutation in the Afp 
gene (AFP-KO) which encodes the major fetal plasma protein alphafetoprotein that binds estradiol with high affinity (Bakker et al., 2007). This finding was quite unexpected since female AFP-KO mice are clearly defeminized with regard to their lordosis behavior (Bakker et al., 2006) as well as their GnRH/Kisspeptin system, i.e., no steroid induced preovulatory LH surges (De Mees et al., 2006; GonzalezMartinez et al., 2008). The observation of a robust male-directed preference in AFP-KO females would thus suggest that the development of mate preferences is postnatally influenced by ovarian estrogens. This finding is in line with our earlier study in female ArKO mice (Bakker et al., 2002b) that suggests feminizing effects of estradiol on the female brain since female ArKO mice showed reduced levels of lordosis behavior as well as no clear mate preferences in adulthood. Taken together, there is still some ambiguity about the role of perinatal estradiol signaling in the development of mate preferences in mice.

Therefore, in the present study, to further investigate the organizational role of prenatal estrogens in mate preferences, we extended our initial study to determine mate preferences in AFP-KO and WT female mice using a different testing paradigm, i.e., a three compartment box in addition to the Y-maze. The rationale for using a three compartment box was to offer the animals different choices between stimulus animals without the need to run from one arm to the other, since preferences measured in the Y-maze may be influenced by differences in exploratory activity of the subjects. Furthermore females were tested under estrous conditions, i.e., following treatment with estradiol and progesterone and not with estradiol alone as was done in our previous study (Bakker et al., 2007). We also determined mate preferences in a group of WT males that were left gonadally intact to determine whether AFP-KO females resembled males or not.

\section{Materials and methods}

\section{Animals}

All breeding and genotyping were performed at the GIGA Neurosciences, University of Liège, Liège, Belgium. In the present study, male and female mice heterozygous for the allele Afptm1lbmm (in the CD1 background strain; Gabant et al., 2002; Bakker et al., 2006) were bred to generate wild-type (WT) and homozygous-null (AFP$\mathrm{KO}$ ) offspring. Mice were genotyped by PCR analysis of tail DNA (for more detailed description, see Bakker et al., 2006, 2007). Subjects were weaned at 21 days and placed into individual cages under a reversed light-dark cycle (12 h:12 h light/dark; $20.00 \mathrm{~h}$ lights on and $8.00 \mathrm{~h}$ lights off) in special light and temperature controlled housing units. Food and water were always available ad libitum.

Stimulus animals (all of the CD1 strain) were gonadally intact males, long-term gonadectomized male ( $g d x)$ and female (ovx) mice, and ovariectomized females brought into behavioral estrus by treatment with estradiol and progesterone. Gonadectomy was performed under general anesthesia after an intraperitoneal injection (i.p.) of a mixture of ketamine $(80 \mathrm{mg} / \mathrm{kg}$ per mouse $)$ and medetomidine (Domitor, Pfizer, $1 \mathrm{mg} / \mathrm{kg}$ per mouse). Mice received atipamezole (Antisedan, Pfizer, $4 \mathrm{mg} / \mathrm{kg}$ per mouse) subcutaneously (s.c.) at the end of the surgery in order to antagonize medetomidineinduced effects, thereby accelerating their recovery. Ovariectomized females that were going to serve as estrous female stimulus were at the same time implanted with a Silastic capsule containing $17 \beta-$ estradiol (diluted 1:1 with cholesterol, for more details see Bakker et al., 2002b).

All experiments were conducted in accordance with the guidelines set forth by the National Institutes of Health "Guiding Principles for the Care and Use of Research Animals" and were approved by the Ethical Committees for Animal Use of the University of Liege.

\section{Behavioral tests}

\section{Mate preferences using the three compartment box}

To assess mate preferences using either visual, auditory, and/or olfactory stimuli, we used a box $(60 \times 13 \times 30 \mathrm{~cm})$ that was divided into three compartments by placing either opaque or transparent partitions. Each compartment was thus $20 \mathrm{~cm}$ in length. The partitions contained perforated holes at a height of $8 \mathrm{~cm}$ to facilitate the diffusion of odors from the two side compartments to the middle compartment. Tests were performed during the dark phase of the light cycle (6 $\mathrm{h}$ after lights out). Animals were habituated to the three compartment box only once on the day before the behavioral experiments by placing them in the middle compartment for $10 \mathrm{~min}$ (with no stimulus animals placed in the two side compartments). On the day of testing, stimulus animals were placed in the two side compartments with their own bedding to make the stimulus as odorous as possible. The subject was then introduced into the middle compartment containing no sawdust, and was observed for $9 \mathrm{~min}$. The time spent poking its nose through the holes of the partition or actively sniffing the bottom of the partition was recorded with a stopwatch. Each test session was divided into $3 \mathrm{~min}$ intervals in order to determine whether investigation times would decrease during the test, since we have previously observed that subjects have the tendency to investigate more during the first minutes of the test. However, since we did not observe any decrease along the test session, we only present the total time spent investigating the two stimuli in the results section.

Thus 24 adult mice (WT: 8 females and 10 males; AFP-KO: 6 females) were used in the present experiments. All females were ovariectomized and implanted in the neck with a $5 \mathrm{~mm}$ long Silastic capsule containing $17 \beta$-estradiol (diluted $1: 1$ with cholesterol) and received an injection of $500 \mu \mathrm{g}$ progesterone $3 \mathrm{~h}$ before each test. Subjects were tested between 10 and 17 weeks of age. All stimulus animals were awake and either presented behind transparent partitions (thus only physical contact was prevented, and mate preferences were based on visual, auditory and olfactory cues) or opaque partitions (to prevent any visual cues). We used the following experimental protocol to test mate preferences: the first series of preference tests were conducted using transparent partitions, thus allowing visual, auditory and olfactory cues. Subjects were first offered a choice between an intact male versus an estrous female. Next, they were offered a choice between an estrous female versus a long-term ovx female, and finally, a choice between an intact male versus a long-term gdx male. The second series of preference tests using opaque partitions, thus only allowing auditory and olfactory cues, was conducted the week after. Again, subjects were first offered a choice between an intact male versus an estrous female, then between an estrous female versus a long-term ovx female, and finally between an intact male versus a long-term gdx male. All tests were conducted on separate days and at least 2 days apart since female subjects were injected with progesterone before each preference test. The position (left versus right compartment) and presentation of the stimulus animals were not randomized to prevent variability due to possible residual odors in the compartments.

\section{Mate preferences using the Y-maze}

Following these tests in the three compartment box, subjects were tested once for their mate preferences in the Y-maze (for a more detailed description of the maze, see Bakker et al., 2002a) with as choice volatile odors from an anesthetized male versus those of an anesthetized estrous female (one AFP-KO female died between the three compartment box and the Y-maze tests). Briefly, before being tested for their mate preferences, all subjects were tested for $5 \mathrm{~min}$ in the Y-maze without any odor stimuli to adapt to the testing apparatus and to determine whether they would develop any side preferences. When subjects were tested for mate preferences using volatile body 
odors (anesthetized stimulus animals) as stimuli, removable perforated opaque Plexiglas doors were placed at the distal end of each arm to separate the goal boxes from the rest of the maze. The time the subject spent sniffing the male and female sides (poking its nose through the holes of the door or actively sniffing the bottom and sides of the door of the goal box) was recorded with a stopwatch for $5 \mathrm{~min}$. The maze was cleaned with Norvanol between trials. The level of anesthesia of the stimulus animals was checked between each trial and stimulus animals were placed regularly on a heating pad to prevent hypothermia.

\section{Statistics}

All behavioral data were analyzed using repeated measures analysis of variance (ANOVA-Statistica 8.0) using stimulus and sex/ genotype as factors. When appropriate, all ANOVAs were followed by Fisher Least Significant Difference post-hoc comparisons adapted for repeated measures ANOVA. Only significant $(P<0.05)$ effects detected by the ANOVAs are presented here.

\section{Results}

Mate preferences using the three compartment box

Table 1 summarizes our predictions regarding mate preferences for the different choice paradigms. These predictions were based on our hypothesis that mate preferences of female AFP-KO mice might resemble those of WT males since they have been exposed to high levels of estrogens before birth. The mate preferences observed in our study are presented in the same table.

\section{Choice: Gonadally intact male versus estrous female}

\section{- Transparent partitions: Visual, auditory, and olfactory stimuli}

As predicted, female WT mice showed a preference for the male whereas female mice showed a preference for the female indicating that mate preferences are influenced by prenatal estrogens. By contrast, male WT male did not show a clear preference (Fig. 1A).

This was confirmed by ANOVA on the time spent investigating the male or female compartment showing a significant interaction between stimulus and sex/genotype $(F(2,22)=8.84, P=0.001)$. Post-hoc analysis indicated that WT females spent more time investigating the side containing the intact male over the one containing the estrous female, whereas AFP-KO females spent more time investigating the side containing the estrous female over the one containing the intact male. Furthermore, WT males spent an equal amount of time investigating the two sides.

\section{- Opaque partitions: Auditory and olfactory stimuli}

As observed with transparent partitions, AFP-KO females showed a preference for the female. By contrast, a preference for the male was less obvious in WT females, whereas WT males, as predicted (Table 1), now showed a preference for the female (Fig. 1B).
This was confirmed by ANOVA on the time spent investigating the male versus the estrous female stimulus showing a significant interaction between stimulus and sex/genotype $(F(2,21)=9.29$, $P=0.001)$. Post-hoc analysis indicated that both AFP-KO females and WT males spent more time investigating the side containing the estrous female compared to the one containing the intact male, whereas WT females spent equivalent amounts of time investigating the two sides.

\section{Choice: Ovx female versus estrous female}

- Transparent partitions: Visual, auditory, and olfactory stimuli

As predicted (Table 1), WT males showed a preference for the estrous female (Fig. 2A). By contrast, AFP-KO females showed a preference for the ovx female whereas WT females showed a preference for the estrous female (Table 2; Fig. 2A). These latter results were not predicted.

ANOVA showed a significant interaction between stimulus and sex/genotype $(F(2,21)=15.24, P<0.001)$. Post-hoc analysis indicated that both WT males and females spent more time investigating the side containing the estrous female over the side containing the ovx female whereas it was reverse with regard to AFP-KO females.

\section{- Opaque partitions: Auditory and olfactory stimuli}

When visual stimuli were blocked by using opaque partitions, none of the experimental groups showed any mate preferences (Fig. 2B). There was a slight trend for a preference for the estrous female in WT mice of both sexes, which was not shown by AFP-KO females.

\section{Choice: Gonadally intact male versus gdx male}

\section{- Transparent partitions: Visual, auditory, and olfactory stimuli}

As predicted (Table 1), WT females showed a preference for the intact male, whereas no clear mate preferences were discerned in AFP-KO females and WT males (Fig. 3A).

ANOVA on the time spent investigating either the intact male or the gdx male showed a significant interaction between stimulus and sex/genotype $(F(2,21)=5.25, P=0.014)$. Post-hoc analysis indicated that WT females spent more time investigating the side containing the intact male over the one containing the gdx male, whereas AFP-KO females and WT males spent equivalent amounts of time investigating both sides.

\section{- Opaque partitions: Auditory and olfactory stimuli}

When the intact male and gdx male were placed behind opaque partitions, very similar results were obtained, i.e., WT females showed a preference for the intact male and AFP-KO females did not show any mate preference (Fig. 3B). By contrast, WT males showed a preference for the intact male.

This was confirmed by ANOVA on the time spent investigating the intact male versus the gdx male showing a significant interaction between stimulus and sex/genotype $(F(2,21)=5.31, P=0.014)$. Post-

Table 1

Theoretical predictions and summary of mate preferences observed under different choice paradigms in the three compartment box. Our working hypothesis was that AFP-KO females might resemble WT males since they are overexposed to estrogens during prenatal development. Overall AFP-KO females never showed the same mate preference as WT females, suggesting that their mate preferences have been defeminized. Abbreviations: int = gonadally intact; est $=$ estrous; ovx $=$ ovariectomized; gdx $=$ gonadectomized; $\mathrm{E} 2+\mathrm{P}=$ estradiol and progesterone treatment; Pred $=$ theoretical predictions; $\mathrm{TP}=$ transparent partitions; $\mathrm{OP}=$ opaque partitions.

\begin{tabular}{|c|c|c|c|c|c|c|c|c|c|c|}
\hline \multirow{3}{*}{ Group } & \multirow{3}{*}{$\begin{array}{l}\text { Hormonal } \\
\text { treatment }\end{array}$} & \multicolumn{9}{|c|}{ Preferences during stimuli presentation } \\
\hline & & \multicolumn{3}{|c|}{$\sigma^{T}$ int Vs. 우 est } & \multicolumn{3}{|c|}{ 우 $_{\text {est }}$ Vs. 우 ovx $_{1}$} & \multicolumn{3}{|c|}{$\sigma^{\top}$ int Vs. $\sigma^{\top} \mathrm{gdx}$} \\
\hline & & Pred & $\mathrm{TP}$ & OP & Pred & $\mathrm{TP}$ & OP & Pred & TP & OP \\
\hline WT 우 & $\mathrm{Ovx} / \mathrm{E} 2+\mathrm{P}$ & $\sigma^{\top}$ int & $\sigma^{\top}$ int & None & None & 우 est & None & $\sigma_{\text {int }}$ & $\sigma^{\top}$ int & $\sigma^{\top}$ int \\
\hline WT ठ ठ & Int & 우 & None & 우 est & 우 est & 우 est & None & None & None & $\sigma^{\top}$ int \\
\hline KO 우 & $\mathrm{Ovx} / \mathrm{E} 2+\mathrm{P}$ & 우est & 우 est & 우est & 우 & 우 ovx & None & None & None & None \\
\hline
\end{tabular}



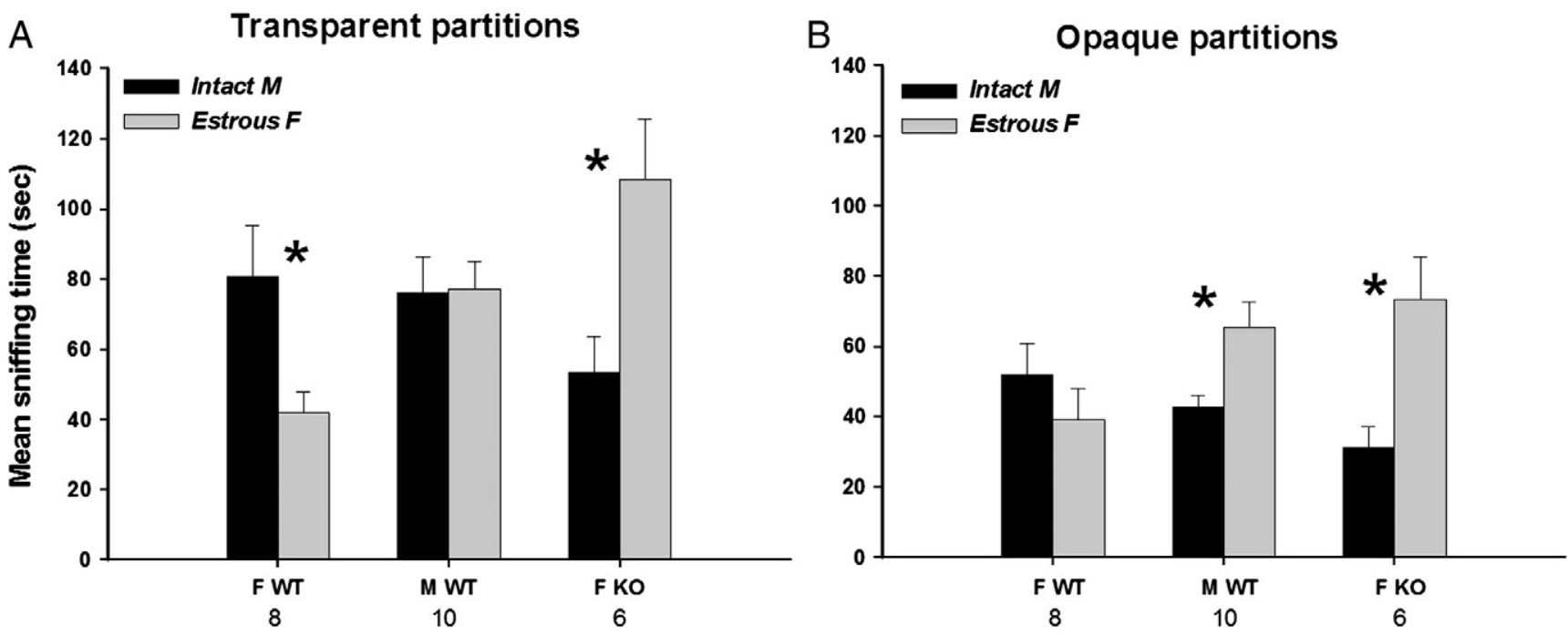

Fig. 1. Mean \pm SEM time spent by AFP-KO females (ovx and E2 + P treated) and WT males (gonadally intact) and females (ovx and E2 + P treated) mice investigating the stimulus animals when given a choice between an intact male and an estrous female behind transparent partitions allowing visual, auditory and olfactory stimulations (A) or behind opaque partitions allowing auditory and olfactory stimulations (B). The number of subjects is indicated below each sex/genotype. ${ }^{*} P<0.05$ post-hoc comparisons between the two presented stimuli.

hoc analysis indicated that WT males and females (almost reached statistical significance; $P=0.059$ ) spent more time investigating the side containing the intact male over the one containing the gdx male (Fig. 3B) whereas AFP-KO females spent equal amounts of time investigating both sides.

\section{Mate preferences using the Y-maze}

In order to determine mate preferences based on olfactory cues only, we tested the subjects in the Y-maze with the choice between volatile odors derived from a gonadally intact male versus those derived from an estrous female. We observed very similar results as in the three compartment box tests. Female WT mice showed a maledirected preference whereas female AFP-KO mice showed a femaledirected preference indicating that mate preferences are influenced by prenatal estrogens (Fig. 4). However, WT males did not show a clear mate preference.

This was confirmed by ANOVA on the time spent investigating volatile body odors from an anesthetized gonadally intact male and an anesthetized estrous female revealing a significant interaction between odor stimulus and sex/genotype $(F(2,20)=5.65$, $P=0.011)$. Post-hoc analysis indicated that WT females spent more time investigating odors from the intact male over those from the estrous female, whereas AFP-KO females spent more time investigating odors from the estrous female. WT males spent equal amounts of time investigating both odor stimuli.

\section{Discussion}

The present study suggests that prenatal exposure to estradiol interferes with some aspects of brain and behavioral sexual
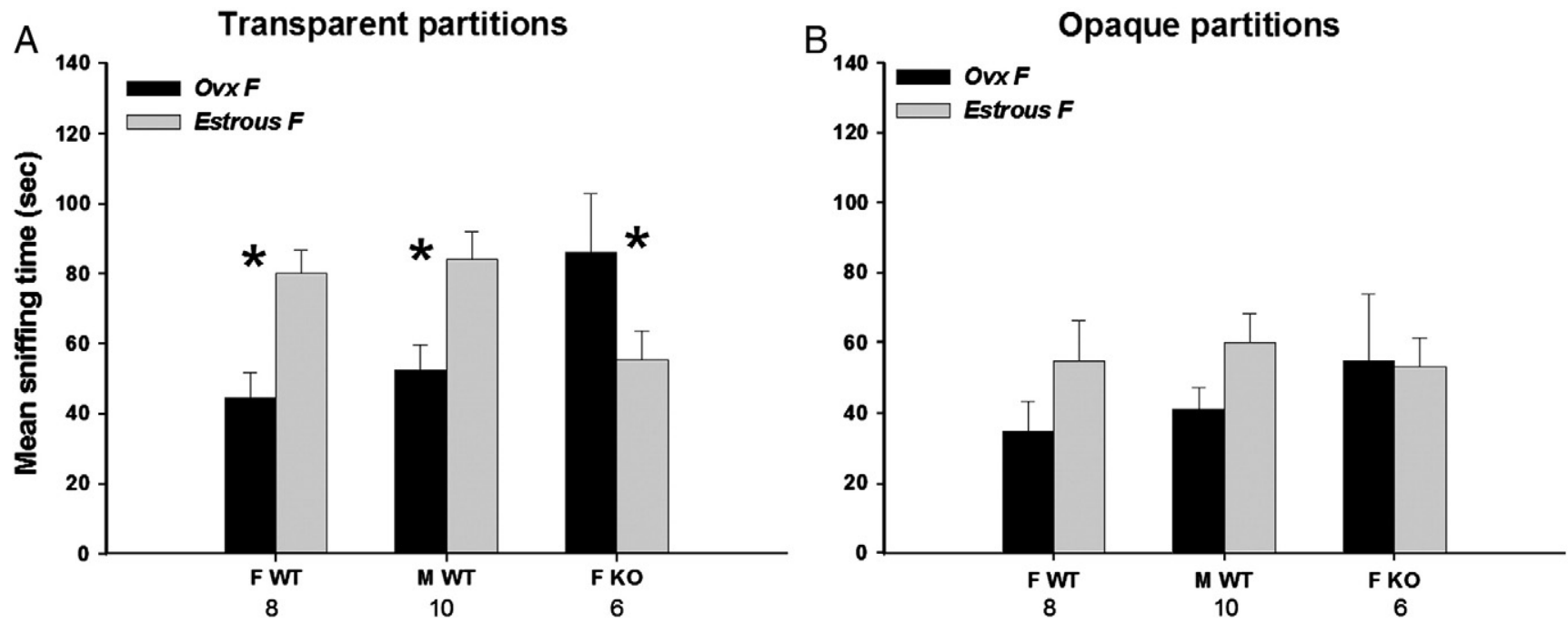

Fig. 2. Mean + SEM time spent by AFP-KO females (ovx and E2 + P treated) and WT males (gonadally intact) and females (ovx and E2 $+P$ treated) mice investigating the stimulus animals when given a choice between a long-term ovariectomized and an estrous female behind transparent partitions allowing visual, auditory and olfactory stimulations (A) or behind opaque partitions allowing auditory and olfactory stimulations (B). The number of subjects is indicated below each sex/genotype. ${ }^{*} P<0.05$ post-hoc comparisons between the two presented stimuli. 

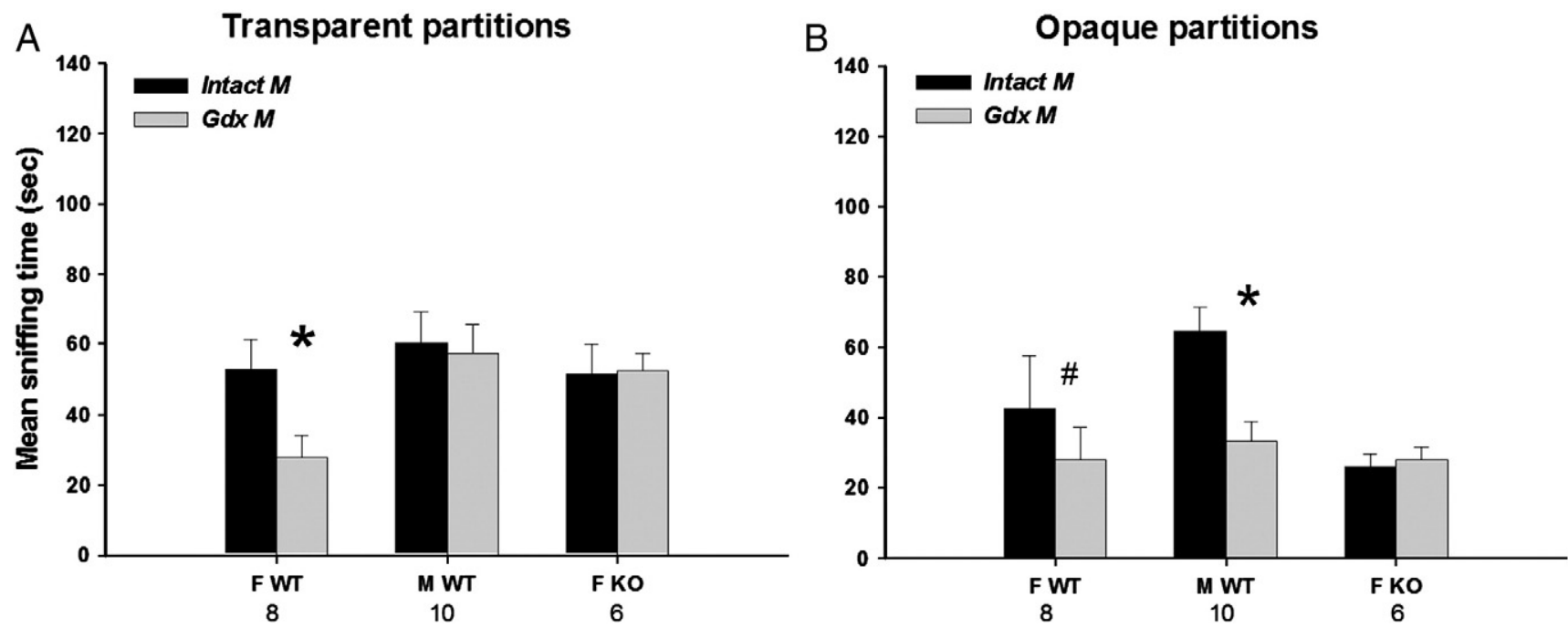

Fig. 3. Mean \pm SEM time spent by AFP-KO females (ovx and E2 + P treated) and WT males (gonadally intact) and females (ovx and E2 + P treated) mice investigating the stimulus animals when given a choice between an intact male and a long-term castrated ( $g d x)$ male behind transparent partitions allowing visual, auditory and olfactory stimulations (A) or behind opaque partitions allowing auditory and olfactory stimulations $(B)$. The number of subjects is indicated below each sex/genotype. ${ }^{*} P<0.05$ post-hoc comparisons between the two presented stimuli and ${ }^{\#} P<0.09$.

differentiation, such as mate preferences. Female mice carrying a targeted mutation in the Afp gene did not show any mate preferences for the intact male under any test condition. The Afp gene encodes the fetal plasma protein, alpha-fetoprotein (AFP), which has a high estradiol binding capacity (Raynaud et al., 1973). Thus AFP-KO females are no longer protected from maternal estrogens during embryonic development (Gabant et al., 2002; Bakker et al., 2006). This result is in line with our earlier observation that AFP-KO females showed no lordosis behavior when paired with a sexually active male, and thus confirms that alpha-fetoprotein protects the brain from defeminizing actions of estradiol on the developing neural mechanisms controlling both proceptive and receptive patterns of feminine sexual behavior. AFP-KO females did not completely resemble WT males in their mate preferences, i.e., they did not show a preference for the estrous female over the ovx female, as was observed in WT males. These results suggest that the sexual differentiation of male-

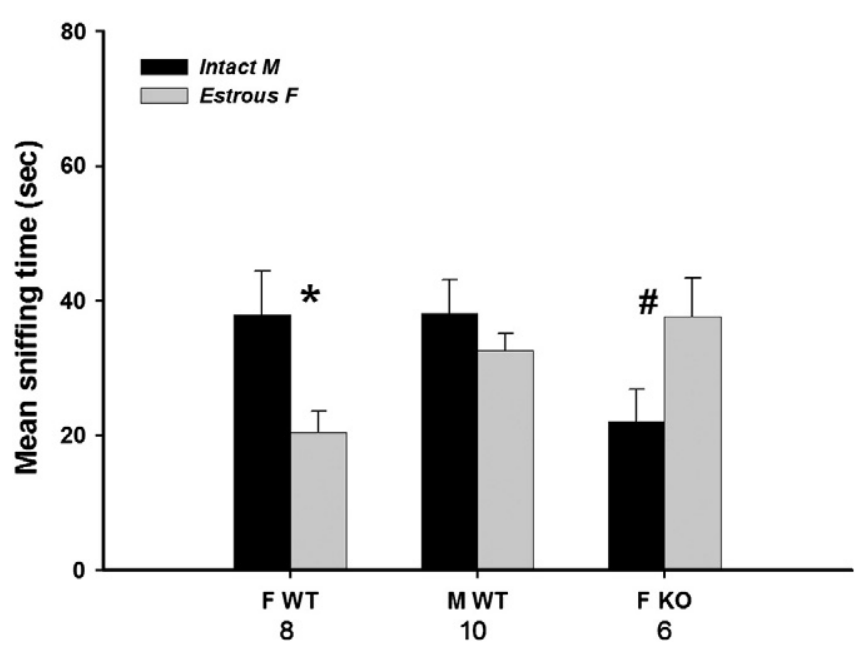

Fig. 4. Mean \pm SEM time that AFP-KO female (ovx and E2 + P treated) and WT male (gonadally intact) and female (ovx and $\mathrm{E} 2+\mathrm{P}$ treated) mice spent investigating volatile body odors when given a choice between intact male odors and estrous female volatile odors in a Y-maze. The number of subjects is indicated below each sex/genotype. ${ }^{*} P<0.05$ and ${ }^{\#} P<0.09$ male versus female odor stimuli. typical aspects of behavior do not solely rely on the action of estradiol during prenatal development, but that it may also require some androgen action. Indeed, Bodo and Rissman (2008) recently observed that female mice treated with a non-aromatizable androgen, i.e., dihydrotestosterone (DHT), on the day of birth, like male controls, showed a preference for female-soiled versus male-soiled bedding. In addition, such DHT-treated female mice showed a preference to investigate an anesthetized female versus an anesthetized male in a Ymaze test. The subjects in this study always had direct access to the olfactory stimuli. Under similar test conditions, we also observed that gonadally intact male ArKO mice resembled WT males in their preference to investigate estrous female bedding over male bedding, when given direct access to both types of bedding in a Y-maze (Bakker et al., 2002a). These results suggest that estrogens are not involved in the sexual differentiation of male-typical and thus female-directed preferences when provided with predominantly non-volatile odors that are contained in soiled bedding. By contrast, when nasal access to the olfactory stimuli was prevented and thus only volatile odors were presented, male ArKO mice failed to show a female-directed preference (Bakker et al., 2002a). This deficit was not corrected by adult treatment with estradiol (Bakker et al., 2004). These results suggest thus a differential role for androgens versus estrogens in the sexual differentiation of male-typical preferences depending on whether the animals have direct access or not to the odor sources. However, we cannot exclude any activational effects of gonadal hormones on mate preferences. Clearly the hormonal status of our subjects, i.e., AFP-KO females and WT males, were not the same at the time of behavioral testing. AFP-KO females were ovariectomized in adulthood and subsequently treated with estradiol and progesterone to induce behavioral estrus, whereas WT males were left gonadally intact. We chose these hormonal regimens in order to test the animals under the most natural hormonal conditions. However, this difference in circulating hormone levels might explain why WT males showed a preference for the estrous female when given the choice between an ovx and an estrous female, whereas female AFP-KO mice did not show this preference. Perhaps if the latter would have been treated with testosterone in adulthood, we would have observed identical mate preferences between WT males and AFP-KO females. Future studies will address this possibility.

Clearly mice can use either volatile or non-volatile odors to seek out a potential mating partner. Traditionally the main olfactory system is 
considered to be important to detect and process volatile odors whereas the accessory olfactory system is thought to primarily detect and process non-volatile odors and to integrate them into the neuroendocrine system leading to behavioral changes. However, lesion studies have shown that the main olfactory system is crucial for mate recognition in both male and female mice whereas the accessory olfactory system seems to be less important (reviewed in Keller et al., 2009). Thus lesions of the main olfactory epithelium (MOE) completely abolished mate preferences (both with and without access to the odor stimuli) whereas lesions of the vomeronasal organ (VNO) only disrupted mate preferences when direct nasal access was provided to the odor stimuli. The latter finding suggests that the VNO and thus investigation of non-volatile odors (as contained in bedding) can contribute to the animals' motivation to approach and investigate potential mates. With regard to the role of gonadal hormones in the sexual differentiation of mate preferences, the accessory olfactory system has sexually dimorphic characteristics (both morphological and functional) at several stages along its projection pathway to the hypothalamus (Bakker et al., 1996a; Bressler and Baum, 1996; Guillamon and Segovia, 1997), and sex steroids upregulate the ability of pheromones to activate neurons throughout this system (Paredes et al., 1998). There is also evidence of sexually differentiated function in the main olfactory system, although these sex differences are less well-described. In habituation/dishabituation studies using gonadectomized mice (Baum and Keverne, 2002; Pierman et al., 2006), females responded more reliably than males to low concentrations of volatile urinary odors from either sex. Furthermore, male ArKO mice resembled WT females in their ability to respond to lower concentrations of male urinary odors (Pierman et al., 2006), raising the possibility that the observed sex difference among WT mice in urine attraction thresholds results from the perinatal actions of estrogen in the male nervous system. However whether these sex differences in urine attraction thresholds and thus main olfactory function relate to the sex differences observed in mate preferences is rather unlikely since these sex differences are already present in the absence of any circulating gonadal hormones, whereas mate preferences clearly need to be activated by gonadal hormones. Thus, how males and females respond differently to olfactory cues may hold key to the neural basis of mate preferences but clearly more studies are needed to unravel the role of both olfactory systems in mate preference and in particular how these neural and behavioral responses are sexually differentiated by gonadal hormones.

In the present study when responding to visual, auditory and/or olfactory stimuli (transparent partitions in the three compartment box), AFP-KO females showed a robust preference for the estrous female over the male indicating that their mate preferences might have been masculinized or defeminized. Likewise, they did not show a preference for the intact male when given a choice between an intact male and a gdx male, whereas WT females clearly preferred the intact male. When we eliminated visual stimuli using opaque partitions to separate the two side compartments from the middle one, we observed some quantitative differences in investigation times of the male and female stimuli, but we observed the same pattern in AFP-KO females, i.e., a preference for the estrous female. Since it has been reported that the CD1 mouse strain (the background strain of the AFPKO mice) show a hereditary deafness (Shone et al., 1991; GMC Coordination Team, Germany, personal communication), we could consider that when using opaque partitions, their mate preference is mostly based on olfactory stimuli; thus, results obtained in the 3 compartment box test with opaque partitions may be compared directly to those obtained in the Y-maze test using anesthetized subjects and thus volatile odors as stimuli. Hence when using the Ymaze, we found the same result, i.e., a female-directed mate preference in AFP-KO females. By contrast, mate preferences were less clear in WT males: they showed no preference for either stimulus animal. This may be due to the fact that WT males are also attracted to male odors for non-sexual reasons, such as aggression and territoriality. However, when AFP-KO females were provided with the choice between an estrous and an ovx female, they failed to show the male pattern, i.e., a preference for the estrous female, indicating that they have not really been masculinized but rather have been defeminized regarding some aspects of brain and behavioral sexual differentiation, such as to approach a sexually active male. However, as mentioned above, it could be possible that when AFP-KO females are tested under testosterone treatment instead of under estrous conditions, they will show the male pattern in mate preferences.

Finally, our current finding of a female- and not a male-directed preference in AFPKO females is in contrast with our earlier result of a male-directed preference in these mice (Bakker et al., 2007). This discrepancy can only be explained by differences in hormonal treatments of the subjects, i.e., estradiol and progesterone in the present study versus estradiol alone in our previous study, underlining thus the importance of hormonal status to observe any genotype and sex differences in mate preferences.

\section{Acknowledgments}

This work was supported by the following grants: Fonds National de la Recherche Scientifique (FNRS) (Mandat d'Impulsion Scientifique F.4502.07) and the NICHD (HD044897) to Dr. Bakker. Dr. Bakker is a Research Associate of the FNRS. We also thank Dr. Mike Baum for commenting on an earlier version of the manuscript.

\section{References}

Bakker, J., 2003. Sexual differentiation of the neuroendocrine mechanisms regulating mate recognition in mammals. J. Neuroendocrinol. 15, 615-621.

Bakker, J., Brand, T., van Ophemert, J., Slob, A.K., 1993. Hormonal regulation of adul partner preference behavior in neonatally ATD-treated male rats. Behav. Neurosci. 107, 480-487.

Bakker, J., Baum, M.J., Slob, A.K., 1996a. Neonatal inhibition of brain estrogen synthesis alters adult neural Fos responses to mating and pheromonal stimulation in the male rat. Neuroscience 74, 251-260.

Bakker, J., Van Ophemert, J., Slob, A.K., 1996b. Sexual differentiation of odor and partner preference in the rat. Physiol. Behav. 60, 489-494.

Bakker, J., Honda, S., Harada, N., Balthazart, J., 2002a. Sexual partner preference requires a functional aromatase (cyp19) gene in male mice. Horm. Behav. 42, 158-171.

Bakker, J., Honda, S., Harada, N., Balthazart, J., 2002b. The aromatase knock-out mouse provides new evidence that estradiol is required during development in the female for the expression of sociosexual behaviors in adulthood. J. Neurosci. 22, 9104-9112.

Bakker, J., Honda, S., Harada, N., Balthazart, J., 2004. Restoration of male sexual behavio by adult exogenous estrogens in male aromatase knockout mice. Horm. Behav. 46 , $1-10$.

Bakker, J., De Mees, C., Douhard, Q., Balthazart, J., Gabant, P., Szpirer, J., Szpirer, C., 2006. Alpha-fetoprotein protects the developing female mouse brain from masculinization and defeminization by estrogens. Nat. Neurosci. 9, 220-226.

Bakker, J., De Mees, C., Szpirer, J., Szpirer, C., Balthazart, J., 2007. Exposure to oestrogen prenatally does not interfere with the normal female-typical development of odour preferences. J. Neuroendocrinol. 19, 329-334.

Baum, M.J., Keverne, E.B., 2002. Sex difference in attraction thresholds for volatile odors from male and estrous female mouse urine. Horm. Behav. 41, 213-219.

Bodo, C., Rissman, E.F., 2008. The androgen receptor is selectively involved in organization of sexually dimorphic social behaviors in mice. Endocrinology 149 $4142-4150$.

Bressler, S.C., Baum, M.J., 1996. Sex comparison of neuronal Fos immunoreactivity in the rat vomeronasal projection circuit after chemosensory stimulation. Neuroscience $71,1063-1072$

De Mees, C., Laes, J.F., Bakker, J., Smitz, J., Hennuy, B., Van Vooren, P., Gabant, P., Szpirer J., Szpirer, C., 2006. Alpha-fetoprotein controls female fertility and prenatal development of the gonadotropin-releasing hormone pathway through an antiestrogenic action. Mol. Cell. Biol. 26, 2012-2018.

Gabant, P., Forrester, L., Nichols, J., Van Reeth, T., De Mees, C., Pajack, B., Watt, A., Smitz J., Alexandre, H., Szpirer, C., Szpirer, J., 2002. Alpha-fetoprotein, the major fetal serum protein, is not essential for embryonic development but is required for female fertility. Proc. Natl Acad. Sci. USA 99, 12865-12870.

Gonzalez-Martinez, D., De Mees, C., Douhard, Q., Szpirer, C., Bakker, J., 2008. Absence of gonadotropin-releasing hormone 1 and Kiss1 activation in alpha-fetoprotein knockout mice: prenatal estrogens defeminize the potential to show preovulatory luteinizing hormone surges. Endocrinology 149, 2333-2340.

Guillamon, A., Segovia, S., 1997. Sex differences in the vomeronasal system. Brain Res. Bull. 44, 377-382. 
Keller, M., Baum, M.J., Brock, O., Brennan, P.A., Bakker, J., 2009. The main and the accessory olfactory systems interact in the control of mate recognition and sexual behavior. Behav. Brain Res. 200, 268-276.

Paredes, R.G., Lopez, M.E., Baum, M.J., 1998. Testosterone augments neuronal Fos responses to estrous odors throughout the vomeronasal projection pathway of gonadectomized male and female rats. Horm. Behav. 33, 48-57.

Pierman, S., Douhard, Q., Bakker, J., 2006. Attraction thresholds and sex discrimination of urinary odorants in male and female aromatase knockout (ArKO) mice. Horm. Behav. 49, 96-104.
Raynaud, J.P., Bouton, M.M., Gallet-Bourquin, D., Philibert, D., Tournemine, C., AzadianBaulanger, G., 1973. Comparative study of estrogen action. Mol. Pharmacol. 9, 520-533.

Shone, G., Raphael, Y., Miller, J.M., 1991. Hereditary deafness occurring in cd/1 mice Hear. Res. 57, 153-156.

Wersinger, S.R., Rissman, E.F., 2000. Oestrogen receptor alpha is essential for femaledirected chemo-investigatory behaviour but is not required for the pheromoneinduced luteinizing hormone surge in male mice. J. Neuroendocrinol. 12 $103-110$ 